\title{
Increasing dopamine levels in the brain improves feedback-based procedural learning in healthy participants: An artificial-grammar-learning experiment
}

\author{
Meinou H. de Vries ${ }^{\mathrm{a}, \mathrm{b}, *}$, Catrin Ulte ${ }^{\mathrm{c}}$, Pienie Zwitserlood ${ }^{\mathrm{d}}$, Barbara Szymanski ${ }^{\mathrm{c}}$, Stefan Knecht ${ }^{\mathrm{c}, e}$ \\ a Department of Psychology and Education, VU University Amsterdam, The Netherlands \\ ${ }^{\mathrm{b}}$ Max Planck Institute for Psycholinguistics, Nijmegen, The Netherlands \\ c Department of Neurology, University of Münster, Germany \\ d Department of Psychology, University of Münster, Germany \\ e Neurocenter, Schön Klinik Hamburg-Eilbek, Germany
}

\section{A R T I C L E I N F O}

\section{Article history:}

Received 9 December 2009

Received in revised form 7 May 2010

Accepted 16 June 2010

Available online 23 June 2010

\section{Keywords:}

Levodopa

Implicit learning

Feedback

Dopaminergic learning

Neuropharmacological modulation

Striatum

\begin{abstract}
A B S T R A C T
Recently, an increasing number of studies have suggested a role for the basal ganglia and related dopamine inputs in procedural learning, specifically when learning occurs through trial-by-trial feedback (Shohamy, Myers, Kalanithi, \& Gluck. (2008). Basal ganglia and dopamine contributions to probabilistic category learning. Neuroscience and Biobehavioral Reviews, 32, 219-236). A necessary relationship has however only been demonstrated in patient studies. In the present study, we show for the first time that increasing dopamine levels in the brain improves the gradual acquisition of complex information in healthy participants. We implemented two artificial-grammar-learning tasks, one with and one without performance feedback. Learning was improved after levodopa intake for the feedback-based learning task only, suggesting that dopamine plays a specific role in trial-by-trial feedback-based learning. This provides promising directions for future studies on dopaminergic modulation of cognitive functioning.
\end{abstract}

(c) 2010 Elsevier Ltd. All rights reserved.

\section{Introduction}

In procedural learning, acquisition of knowledge occurs gradually, and is based on an ongoing presentation of multiple stimuli and responses (thus, involving trial-by-trial feedback), exemplified by the acquisition of motor skills or language rules (Ullman, 2004). Recently, procedural learning has attracted a substantial amount of interest, emerging from neuro-imaging, animal and lesion studies, with the consensus that the basal-ganglia system and the prefrontal regions to which it projects, subserve as a neural correlate for this type of learning (Shohamy, Myers, Kalanithi, \& Gluck, 2008; Ullman, 2004). Whereas only few studies have tested healthy participants' striatal involvement in trial-by-trial feedback learning, several patient studies have shown that basal-ganglia disorders particularly disrupted feedback-based procedural learning, as assessed by the Weather Prediction Task, where participants had to learn probabilistic cue-outcome associations over many trials(Knowlton, Mangels, \& Squire, 1996; Knowlton, Squire, et al.,

\footnotetext{
* Corresponding author at: Department of Psychology and Education, VU University Amsterdam, Van der Boechorststraat 1, 1081 BT Amsterdam, The Netherlands Tel.: +31 205983 836; fax: +3120598 8745 .

E-mail address: mh.de.vries@psy.vu.nl (M.H. de Vries).
}

1996). This finding has been corroborated by an fMRI study with healthy participants (Poldrack et al., 2001), showing engagement of the basal ganglia in a trial-by-trial feedback-based learning task. Interestingly, this activation was decreased when the same task was learned without feedback, although performance levels were similar. Since fMRI methods cannot demonstrate the necessity of the striatum in feedback-based learning, Shohamy, Myers, Onlaor, and Gluck (2004) replicated this experiment in Parkinson's disease (PD) patients, typically suffering from a loss of nigro-striatal dopamine neurons that disrupts striatal functioning (Agid, JavoyAgid, \& Ruberg, 1987). As expected, they were only impaired on the feedback-based task, but not on the non-feedback version of the same task. Another way of assessing procedural learning is through the artificial-grammar-learning (AGL) task, where participants learn a complex grammar after being exposed to positive exemplars only, hence, no feedback is involved. Here, PD patients were not impaired (Reber \& Squire, 1999). Smith and McDowall (2006) manipulated the AGL task such that participants had to learn through trial-by-trial feedback, and found that PD patients are selectively impaired in a feedback-based version of the AGL task (Smith \& McDowall, 2006). A consistent result throughout the literature is thus, that PD patients are impaired when learning occurs through feedback, but not when learning occurs through merely observing exemplars of a specific category (i.e., observational learning). 
In the present study, we wanted to show a direct relationship between the dopamine system and feedback-based procedural learning in healthy adults, which, to our knowledge, has not been demonstrated before. To do so, we implemented two complex artificial-grammar-learning experiments, one with and one without feedback, and administered levodopa. Note that we did not intend to draw any conclusions about the role of the striatum in procedural learning. Rather, we hypothesized that increasing dopamine levels in the brain would affect learning success in the feedback-based version, but not in the observational learning version.

The outputs from the basal ganglia to the cortex are affected by dopaminergic projections from the substantia nigra to the neostriatum. Across species, the most common finding is that the dopaminergic neurons in the substantia nigra play a major role in the reward-based learning functions of the basal ganglia (Doya, 2000; Packard \& Knowlton, 2002). In the current study, we set out to modulate the outputs from the basal ganglia to the frontal cortex, and hence its functioning, by increasing dopamine levels in the brain. We tested healthy participants who were administered levodopa, a precursor of dopamine, or a placebo substance. Levodopa has been demonstrated before to enhance cognitive functioning and motor learning in healthy participants (Floel et al., 2008; Knecht et al., 2004). Phasic dopamine is crucially involved in learning success (Fiorillo, 2004), whereas dopamine agonists, which affect tonic dopamine levels, lead to learning impairment (Breitenstein et al., 2006). Understanding how levodopa affects learning and memory is therefore not only of great interest from a clinical perspective, but also for gaining more insight into the neural correlates of the role of dopamine in learning and memory (Shohamy, Myers, Geghman, Sage, \& Gluck, 2006; Shohamy et al., 2008).

We implemented two complex artificial-grammar-learning experiments. In the first, participants had to merely observe a large amount of stimuli, all conforming to an underlying structure, i.e., grammar. In a subsequent classification test, they then had to decide whether or not novel stimuli conformed to the grammar. In the second task, participants were given performance feedback after each trial, such that they were only able to learn the grammar through the delivered feedback.

\section{Methods}

\subsection{Participants and procedure}

In a randomized, double-blind, placebo-controlled between-group study with two groups of twenty participants, we investigated the influence of taking $100 \mathrm{mg}$ levodopa in combination with $25 \mathrm{mg}$ of the decarboxylase inhibitor carbidopa. The placebo group received a standard placebo substance of $99.5 \%$ mannitol and $.5 \%$ erosil. All medication was produced in identical capsules. Medication was given $60 \mathrm{~min}$ prior to participation in the experiment, to achieve optimal blood plasma levels. The timeline of the experiment is depicted in Fig. 1. Because of the doubleblind nature of the study, we were unable to balance participants over testing days such that all participants of both groups were assessed with learning without feedback on Day 1, and learning with feedback on Day 2. Importantly, levodopa has a plasma-half-life of $60 \mathrm{~min}$, and at a dose of $100 \mathrm{mg}$, does not induce noticeable long duration responses (Dethy et al., 1997; Zappia et al., 1999). Performance on Day 2 is thus unlikely to be affected by the medication intake on Day 1. The Human Subject Committee of the University of Münster approved the study and it was performed according to the 1964 Declaration of Helsinki.

Forty right-handed male participants (mean age 24.2, SD 3.1) were recruited; all were native German speakers, raised in Germany and weighed $65-95 \mathrm{~kg}$. They did not have any history of neurological, psychiatric or cardiological disorders, chronic, acute diseases, or known drugs allergies, intake of drugs affecting the central nervous system up to 2 weeks before study participation, consumption of recreational drugs as assessed by a urinary drugs test, and a daily use of more than 15 cigarettes, 6 cups of coffee and $50 \mathrm{gr}$ alcohol. All participants gave written informed consent and were informed that they could withdraw from the experiment at any time without any reason.

\subsection{Neuropsychological test battery}

All participants were tested with a neuropsychological test battery, including tests of general intellectual performance, working memory and attention, digit spans and sensation seeking. Blood pressure and the PANAS (Positive and Negative Affective Schedule) were assessed before and after each session.

\subsection{Experiment 1: observational artificial-grammar-learning}

This experiment consisted of an acquisition phase and a classification phase. The stimuli were generated from a finite-state grammar (Reber, 1967), depicted in Fig. 1. We generated 150 grammatical $(G)$ strings from this grammar, with a string length of 5-12 symbols. 100 strings were used for the acquisition phase and 50 for the classification phase (together with 50 non-grammatical (NG)). The strings in the classification phase matched the strings in the acquisition phase in relative chunk strength, such that participants could not discriminate grammatical from non-grammatical strings based on superficial features such as bi- and tri-grams that frequently occurred during training (cf. Knowlton \& Squire, 1996). Non-grammatical strings were derived by a switch of letters in two non-terminal positions.

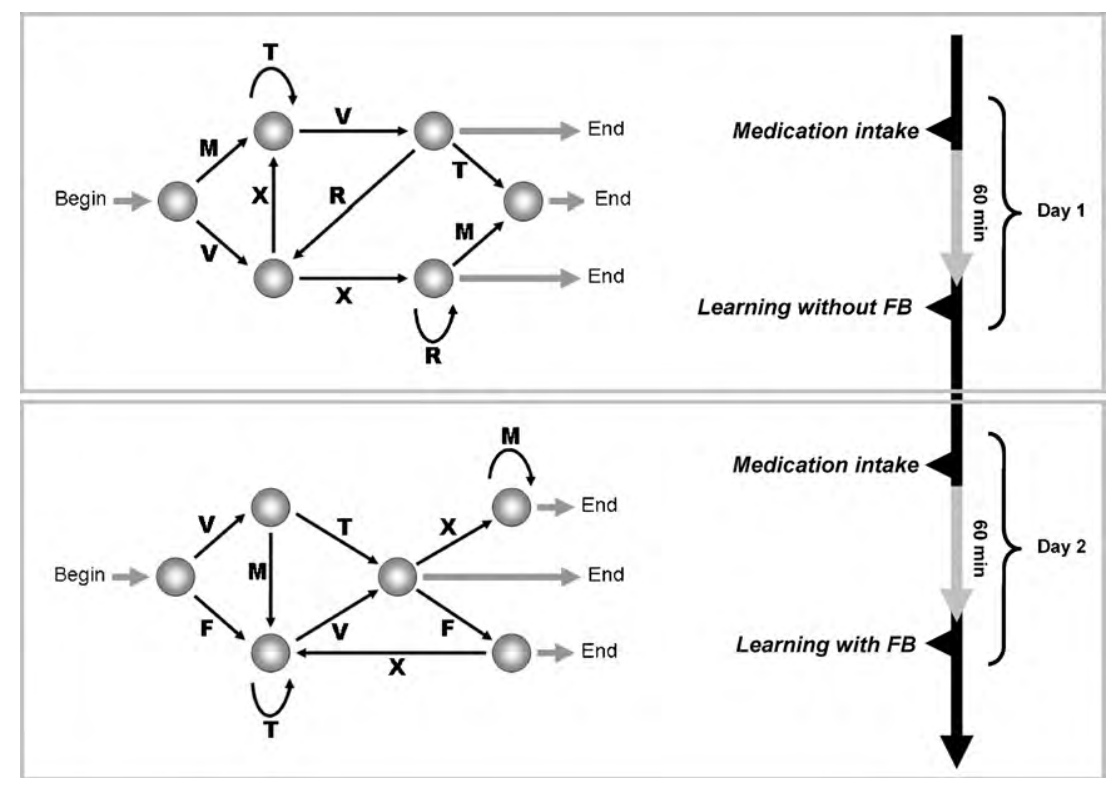

Fig. 1. The grammars used for the non-feedback and feedback-based artificial-grammar-learning experiment, and the experimental timeline. 
In the training phase, participants were told that they participated in a shortterm-memory task, and were presented with one letter string at a time on a computer screen. They were instructed to closely pay attention, and after the string had disappeared, to immediately recall it as accurately as possible, by retyping the string on the key-board. Timing was self-paced and the participants could correct themselves by using the back-space button. Each string was presented for $5 \mathrm{~s}$ and was centrally placed on a computer screen. Only grammatical exemplars were given and no performance feedback was provided. For the classification task ( $\sim 15 \mathrm{~min})$, the participants were instructed to classify novel strings as grammatical or nongrammatical by pressing the corresponding buttons. They were told to respond as accurately and as quickly as possible, relying on their intuition. The whole experiment lasted $\sim 50 \mathrm{~min}$.

\subsection{Experiment 2: feedback-based artificial-grammar-learning}

The grammar used to generate the strings for Experiment 2 is displayed in Fig. 1 and was equally complex to the grammar in Experiment 1 with respect to number of transitions and elements.

200 grammatical strings were generated with a string length of 5-12 symbols. Of these, 100 strings were selected to serve as non-grammatical strings, created by a switch of letters in two non-terminal positions. Of all 200 strings, two blocks of 100 trials (50 non-grammatical) were created, such that we could analyze learning success over time-assuming that learning would take place only after substantial familiarization. Moreover, the amount of trials in one testing block was now equal in size to the testing block in Experiment 1.

Letter strings were presented for $5 \mathrm{~s}$, placed centrally on the computer screen. The response-time window was $3 \mathrm{~s}$. Participants responded by pressing one of the two buttons, deciding whether the string that they had just seen was grammatical or non-grammatical. Following a response, the computer provided feedback, displaying either a smiley (correct response), or a whiney (incorrect response) for $2 \mathrm{~s}$.

Participants were instructed the letter strings they would see were generated by a very complex rule system, or grammar. They would be asked to classify each string as grammatical or non-grammatical, guessing in the beginning, but using the feedback on each trial to determine whether the letter strings in the subsequent trials were grammatical or not. It was made clear to the participants that initially they would have the feeling that they were guessing, but that their performance would gradually improve as the experiment progressed (Smith \& McDowall, 2006 used a similar approach). As in Experiment 1, they were instructed to base their judgments on their intuition and respond as quickly and accurately as possible. The participants were told clearly that the grammar of this experiment was unrelated to Experiment 1.

For both experiments, we used the Presentation software (www.neuro-bs.com) for programming.

\section{Results}

From the 40 participants, we excluded two who performed below chance level $(<50 \%)$, as well as two weight-matched controls, such that two equally sized groups of 18 participants each remained.

\subsection{Neuropsychological tests}

The neuropsychological tests did not reveal any group differences. Furthermore, a repeated measures ANOVA Group (Levodopa vs. Placebo $\times$ Time (Before vs. After the experiment) revealed no group differences that could be explained by different mood ratings as assessed by the PANAS rating scale.

\subsection{Experiment 1: observational artificial-grammar-learning}

For the acquisition phase, there was no significant difference $(p=.341)$ in correct recalls (Levodopa group: 47; Placebo group: 41 ). For the test phase, we calculated the endorsement rates (test items perceived as grammatical, i.e., the hits and false alarms) for both groups using standard detection theory (Hochhaus, 1972). We conducted a Group (Placebo vs. Levodopa) $\times$ Grammaticality (Grammatical items vs. Non-grammatical items) ANOVA. This yielded a main effect for Grammaticality, with $F(1,16)=5.65$ and $p<.0001$, indicating that learning took place in both groups. There was no significant effect for Group, nor a significant interaction of Group $\times$ Grammaticality. See Fig. 2 for the endorsement rates of both groups. There were no group differences in reaction times whatsoever.

\subsection{Experiment 2: feedback-based artificial-grammar-learning}

A repeated measures ANOVA Group (Placebo vs. Levodopa) $\times$ Block (1 vs. 2$) \times$ Grammaticality (Grammatical vs. Nongrammatical items), with endorsement rate as dependent variable, was conducted over the two blocks. There was a main effect for Grammaticality $(F(1,16)=18.77, p=.001)$, and an interaction of Group $\times$ Block $(F(1,16)=5.95, p<.05)$, indicating that only the Levodopa group demonstrated significant learning improvement. Posthoc $t$-tests revealed that, for the second block, the Levodopa group showed a significantly higher hit rate, with $\mathrm{t}(32)=2.07$ and $p<.05$. See Fig. 2 for the endorsement rates in the second block. There were no significant differences in reaction time whatsoever.

Additionally, we compared endorsement rates very early vs. very late in learning, by conducting an ANOVA with the factors Grammaticality (Grammatical vs. Non-grammatical items) $\times$ Group (Placebo vs. Levodopa) $\times$ Trial block (trials 1-25 vs. trials 176-200), which revealed significant interactions of Grammaticality $\times$ Trial block $(F(1,16)=14.86, p=.001)$ and Group $\times$ Trial block $(F(1,16)=5.06, p<.05)$, indicating that $(1)$ sensitivity to test items improved over the two blocks, and (2) that learning improve-

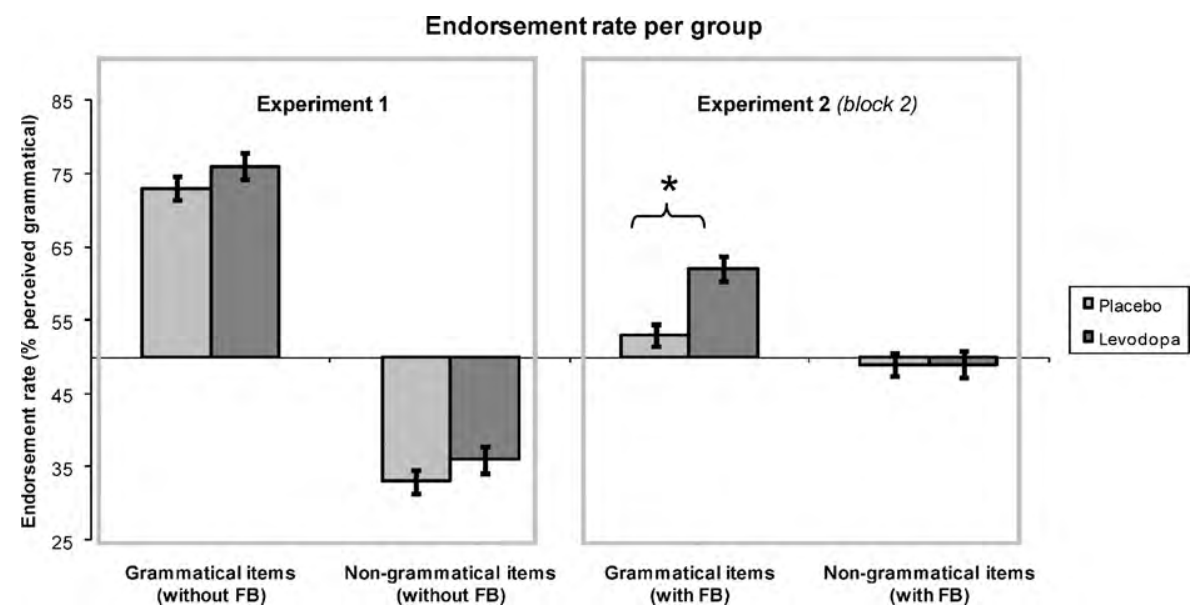

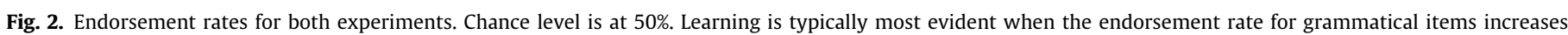
(number of hits) and for non-grammatical items (number of false alarms) decreases. Error bars indicate standard error of the mean. 
ment was specific for the Levodopa group. Again, significance was driven by a substantially increased number of correctly classified items in the Levodopa group.

\section{Discussion}

\subsection{Main findings}

The Levodopa group showed significantly improved learning compared to the Placebo group, but only for the feedback-based artificial-grammar-learning task. This improvement depends on the medication and is mainly driven by a significantly better ability to correctly identify grammatical items later in learning, as compared to the Placebo group.

\subsection{Trial-by-trial feedback learning and the dopamine system}

Feedback modulates dopamine release in the basal ganglia. A healthy range of dopamine bursts during feedback may lead to the acquisition of stimulus-reward response associations (Frank, 2005). Previous studies suggest that positive and negative feedback have opposing effects on dopamine release, which in turn modulates synaptic plasticity and therefore supports learning (Frank, 2005; Frank, Seeberger, \& O’Reilly, 2004). Studies in both healthy participants and patients have shown that the basal ganglia and its dopaminergic projections indeed play a major role in trialby-trial feedback-based learning situations (Knowlton, Mangels, et al., 1996; Poldrack et al., 2001; Shohamy et al., 2004). Our results complement these results, by corroborating the necessity of dopaminergic signaling in trial-by-trial feedback-based learning also in healthy participants.

In our study, performance was poorer for feedback-based learning compared to observational learning for both groups. Similar results were reported by Wilkinson, Lagnado, Quallo, and Jahanshahi (2008), who found that, on the Weather Prediction Task, both PD patients and healthy controls performed worse on the feedback-based version of the task compared to the observational version. People might find it easier to learn information that is presented in an observational than in a feedback-based manner (Wilkinson et al., 2008). An explanation for such a preference is that observational learning provides a situation where errorless learning takes place, leading to greater accuracy than errorful conditions (Baddeley \& Wilson, 1994; Wilkinson et al., 2008).

Alternatively, Maxwell, Masters, Kerr, and Weedon (2001) suggested that reducing errors (i.e., learning with positive exemplars only) minimizes the involvement of explicit hypothesis testing. Performance on AGL tasks is known to decrease under explicit hypothesis testing, as the material is too complex to acquire when intentionally attempting to learn a pattern - indeed, in such situations, AGL performance might be hindered (e.g. Howard \& Howard, 2001; Reber, 1967). Observational learning therefore might be beneficial in situations where the to-be-learned material is relatively complex, as was the case in our two AGL experiments, which can explain the generally poorer performance on feedback-based learning. The finding that the Levodopa group scored better in the feedback-based learning situation could imply that they used feedback more effectively than the Placebo-group.

\subsection{Artificial-grammar-learning and the striatum}

No consistent learning impairments in artificial-grammarlearning tasks in patients suffering from basal-ganglia diseases have been found (Knowlton, Squire, et al., 1996; Peigneux, Meulemans, Van der Linden, Salmon, \& Petit, 1999; Reber \& Squire, 1999; Witt, Nuehsman, \& Deuschl, 2002), nor a dopaminergic medication effect (Witt et al., 2002). This can be explained by the nature of the task: nearly all studies implemented the "traditional" artificial-grammar-learning task (Reber, 1967), which is similar to our observational artificial-grammar-learning task. Here, learning occurs through exposing individuals to a large amount of stimuli, all conforming an underlying structure, and instructing them to memorize the stimuli. Only in a later stage are they asked to make judgments about these regularities. The critical difference with trial-by-trial feedback-based learning is that the latter requires the participant to learn to associate various stimuli with behavioural responses delivered by performance feedback (Reber \& Squire, 1999). Only this type of learning seems to be impaired in diseases of the basal ganglia. Smith and McDowall (2006) conducted a similar trial-by-trial feedback-based artificial-grammar-learning experiment, and they indeed found that PD patients, compared to healthy controls, were impaired in learning the grammar.

\subsection{Procedural learning and language learning}

Ullman (2004) proposed that the basal ganglia play a crucial role in the "procedural features" of language, such as grammar acquisition, as opposed to the medial temporal lobe in the "declarative features" of language, such as semantic memory. The basal ganglia are involved in aspects of selection and maintenance in working memory of structured elements in complex linguistic representations, and in the learning of rules over these representations (Ullman, 2004). Grammar is learned and processed by channels in the basal ganglia that run throughout the basal ganglia to the thalamus and frontal cortex (Ullman, 2004). Furthermore, the frontal areas to which the basal ganglia project, specifically Broca's area, the supplementary motor area (SMA) and the pre-SMA, also subserve aspects of grammar (Ullman, 2004). Our findings however suggest that not all procedural learning involves dopaminergic projections, but that it is specific to feedback-based procedural learning only.

\subsection{Future perspectives}

We are the first to show that increasing dopamine levels in the brain improves feedback-based procedural learning in healthy adults. The involvement of dopamine in such learning, as assessed in PD patients in the previous literature, was still unclear, since dopaminergic medication may affect cognitive function differentially due to an "overdose" effect (Cools, Barker, Sahakian, \& Robbins, 2001). Specifically, the ventral striatum, involved in feedback-based procedural learning, is affected by PD only at a relatively late stage. Dopaminergic medication in an early stage of PD might therefore "overdose" the ventral striatum, resulting in impaired performance on the "reversal learning" task (Cools et al., 2001; Cools, Barker, Sahakian, \& Robbins, 2003).

We suggest that our results are potentially relevant to patients with ventral striatal lesions, in situations where learning occurs through stimulus-response associations. Future studies should further investigate the possibility of enhancing cognitive functioning through neuropharmacological modulation.

\section{Conclusion}

We found improved learning for the Levodopa group as compared to the control group, but only for the artificial-grammarlearning task where learning occurred through trial-by-trial feedback. This is consistent with evidence on procedural learning tasks that trial-by-trial feedback learning is dependent on the basal ganglia. PD patients have been found to be substantially impaired on this kind of learning. Our experiments on healthy subjects support and complement the previous findings that dopamine levels in the brain indeed play a role in feedback-based procedural learning. 
Furthermore, our results speculate about which types of learning might depend on the basal-ganglia system, and are promising for research on dopaminergic modulation of cognitive functioning.

\section{Acknowledgements}

This research was funded by the BMBF (Federal Ministery of Education and Research, Germany), and the EU 6th Framework Program Marie Curie Research and Training Network: Language and the Brain, awarded to Meinou de Vries and Stefan Knecht. We thank Elina Sakellaridou and Julia Reinholz for their valuable help with the experiments, Caterina Breitenstein, Bianca Drager and Sandra Kamping for their contributions to project preparations, and two anonymous reviewers for their insightful comments.

\section{References}

Agid, Y., Javoy-Agid, F., \& Ruberg, M. (1987). Biochemistry of neurotransmitters in Parkinson's disease. Movement Disorders, 2, 166-230.

Baddeley, A., \& Wilson, B. A. (1994). When implicit learning fails: Amnesia and the problem of error elimination. Neuropsychologia, 32(1), 53-68.

Breitenstein, C., Korsukewitz, C., Floeel, A., Kretzschmar, T., Diederich, K., \& Knecht, S. (2006). Tonic dopaminergic stimulation impairs associative learning in healthy subjects. Neuropsychopharmacology, 31, 2552-2564.

Cools, R., Barker, R. A., Sahakian, B. J., \& Robbins, T. W. (2001). Enhanced or impaired cognitive function in Parkinson's disease as a function of dopaminergic medication and task demands. Cerebral Cortex, 11, 1136-1143.

Cools, R., Barker, R. A., Sahakian, B. J., \& Robbins, T. W. (2003). L-Dopa medication remediates cognitive inflexibility, but increases impulsivity in patients with Parkinson's disease. Neuropsychologia, 41(11), 1431-1441.

Dethy, S., Laute, M. A., Van Blercom, N., Damhaut, P., Goldman, S., \& Hildebrand, J. (1997). Microdialysis-HPLC for plasma levodopa and metabolites monitoring in parkinsonian patients. Clinical Chemistry, 43(5), 740-744.

Doya, K. (2000). Complementary roles of basal ganglia and cerebellum in learning and motor control. Current Opinion in Neurology, 10, 732-739.

Fiorillo, C. D. (2004). The uncertain nature of dopamine. Molecular Psychiatry, 9, $122-123$.

Floel, A., Garreaux, G., Xu, B., Breitenstein, C., Knecht, S., Herscovitch, P., et al. (2008). Levodopa increases memory encoding and dopamine release in the striatum in the elderly. Neurobiology of Aging, 29(2), 267-279.

Frank, M. J. (2005). Dynamic dopamine modulation in the basal ganglia: A neurocomputational account of cognitive deficits in medicated and nonmedicated parkinsonism. Journal of Cognitive Neuroscience, 17(1), 51-72.

Frank, M. J., Seeberger, L. C., \& O'Reilly, R. C. (2004). By carrot or by stick: Cognitive reinforcement learning in Parkinsonism. Science, 306(5703), 1940-1943.

Hochhaus, L. (1972). A table for the calculation of d' and BETA. Psychological Bulletin, 77(5), 375-376.
Howard, D. V., \& Howard, J. H. (2001). When it does hurt to try: Adult age differences in the effects of instructions on implicit pattern learning. Psychonomic Bulletin and Review, 8(4), 798-805.

Knecht, S., Breitenstein, C., Bushuven, S., Wailke, S., Kamping, S., Floeel, A., et al. (2004). Levodopa: Faster and better word learning in normal humans. Annals of Neurology, 56(1), 20-26.

Knowlton, B. J., Mangels, J. A., \& Squire, L. R. (1996). A neostriatal habit learning system in humans. Science, 273, 1399-1402.

Knowlton, B. J., \& Squire, L. R. (1996). Artificial grammar learning depends on implicit acquisition of both abstract and exemplar-specific information. Journal of Experimental Psychology: Learning Memory and Cognition, 22, 169-181.

Knowlton, B. J., Squire, L. R., Paulsen, J., Swerdlow, N., Swenson, M., \& Butters, N. (1996). Dissociations within nondeclarative memory in Huntington's disease. Neuropsychology, 10, 538-548.

Maxwell, J. P., Masters, R. S. W., Kerr, E., \& Weedon, E. (2001). The implicit benefit of learning without errors. The Quarterly Journal of Experimental Psychology Section A, 54(4), 1049-1068.

Packard, M., \& Knowlton, B. (2002). Learning and memory functions of the basal ganglia. Annual Review of Neuroscience, 25, 563-593.

Peigneux, P., Meulemans, T., Van der Linden, M., Salmon, E., \& Petit, H. (1999). Exploration of implicit artificial grammar learning in Parkinson's disease. Acta Neurologica Belgica, 99, 107-117.

Poldrack, R. A., Clark, J., Pare-Blagoev, E. J., Shohamy, D., Moyano, J. C., Myers, C., et al. (2001). Interactive memory systems in the human brain. Nature, 414, 546-550.

Reber, A. S. (1967). Implicit learning of artificial grammars. Journal of Verbal Learning E'Verbal Behavior, 6, 855-863.

Reber, P. J., \& Squire, L. R. (1999). Intact learning of artificial grammars and intact category learning by patients with Parkinson's disease. Behavioural Neuroscience, 113(2), 235-242.

Shohamy, D., Myers, C. E., Geghman, K. D., Sage, J., \& Gluck, M. A. (2006). L-Dopa impairs learning, but spares generalization, in Parkinson's disease. Neuropsychologia, 44(5), 774-784.

Shohamy, D., Myers, C. E., Kalanithi, J., \& Gluck, M. A. (2008). Basal ganglia and dopamine contributions to probabilistic category learning. Neuroscience and Biobehavioral Reviews, 32, 219-236.

Shohamy, D., Myers, C. E., Onlaor, S., \& Gluck, M. A. (2004). Role of the basal ganglia in category learning: How do patients with Parkinson's disease learn? Behavioral Neuroscience, 118(4), 676-686.

Smith, J. G., \& McDowall. (2006). When artificial grammar acquisition in Parkinson's disease is impaired: The case of learning via trial-by-trial feedback. Brain Research, 1067(1), 216-228.

Ullman, M. T. (2004). Contributions of memory circuits to language: The declarative/procedural model. Cognition, 92(1-2), 231-270.

Wilkinson, L., Lagnado, D. A., Quallo, M., \& Jahanshahi, M. (2008). The effect of feedback on non-motor probabilistic classification learning in Parkinson's disease. Neuropsychologia, 46, 2683-2695.

Witt, K., Nuehsman, A., \& Deuschl. (2002). Intact artificial grammar learning in patients with cerebellar degeneration and advanced Parkinson's disease. Neuropsychologia, 40(9), 1534-1540.

Zappia, M., Bosco, D., Plastino, M., Nicoletti, G., Branca, D., Oliveri, R. L., et al. (1999). Pharmacodynamics of the long-duration response to levodopa in PD. Neurology, 53(3), 557-560. 